\title{
Cinco de Mayo: la fiesta de los EE.UU.
}

\section{Porfirio López}

Hay cosas que sentimos en la piel, otras que vemos con los ojos, otras que nomás nos laten en el corazón. - Carlos Fuentes

El Cinco de Mayo se conmemora la Batalla de Puebla (1862), acontecimiento en el que el pueblo mexicano derrotó al ejército francés, el cual se denominaba como el gran ejército del mundo. Aunque este suceso se conmemora en los Estados Unidos en áreas que son relativamente habitadas por mexicoamericanos, también hay otros lugares en los que predominan otros latinos (cubanos, nicaragüenses, argentinos etc.) que también lo celebran. El Cinco de Mayo simboliza un orgullo y motivo para el progreso de los mexicanos. Para los que no tienen un buen conocimiento, el Cinco se caracteriza por ser una pachanga llena de fiesta, banderas en movimiento, comida mexicana y la repetición de "El Rey" de Vicente Fernández por el radio. Pero es importante analizar el significado y la importancia de este día festivo. Este ensayo trata de dar una reconstrucción del significado del Cinco de Mayo para los latinos en EE.UU. Pero mucho más antes, debemos dar un vistazo hacia el México de ayer donde todo comenzó.

\section{E1 inicio}

La batalla de Puebla el 5 de Mayo de 1862 da inicio al sentimiento nacional iniciado hacía varios años atrás en el territorio de Nueva España (México). Tras varios años de lucha, la independencia de Nueva España llegó en 1821 con el Plan de Iguala proclamado el 24 de febrero. Este Plan implicaba una especie de igualdad entre los criollos/mestizos y los peninsulares. Después de la Independencia, hubo desacuerdos entre los mexicanos. Por un lado, los conservadores querían un gobierno centralista y una unión entre el estado y la iglesia, pero los liberales (rancheros, mineros, artesanos, maestros, artistas, etc.) se opusieron a esta propuesta de nación. Más de 30 líderes fueron presidentes de México pero no fue hasta la Guerra de Reforma (1857-1860) que una posible intervención extranjera fuera pensada por parte de los conservadores como la solución. Fue así como la guerra entre liberales y conservadores causó una gran deuda con los países (España, Gran Bretaña y Francia) que ayudaron financiar a los liberales. México estaba en un gran momento de crisis. A la vez que no tenían estructura fija en el gobierno, debido a los desacuerdos entre el 
gobierno, no había dinero lo cual llevó a que el Presidente Benito Juárez otorgara una suspensión de pago a los países extranjeros por dos años hasta que hubiera estabilidad económica. E1 25 de enero de 1862 Benito Juárez intentó una vez más llegar a un acuerdo con los países para evitar un choque y llegaron a firmar Los Preliminares de la Soledad. Este tratado declaró que los países europeos tenían garantizados el pago de deudas. España y Gran Bretaña partieron mientras Francia, con la ayuda de los conservadores eligió mantenerse en México hasta que llegaran sus refuerzos a la costa de Veracruz, lo cual ocurrió el 20 de abril cuando el General Charles Latrille comenzó la invasión a México acompañado de más de 6,000 soldados. El general mexicano Ignacio Zaragoza, quien nació en Goliad, Texas, en 1829, era el Comandante de Cuerpo del Ejército de Oriente y llegó a la Ciudad de Puebla el 3 de mayo para prevenir la toma de la Ciudad de México por los franceses. Cuando Zaragoza se dio cuenta que los conservadores se habían unido a los franceses, ordenó a un buena parte de su ejército que impidiera el avance de las fuerzas conservadoras, quienes especulaban que llegarían de una dirección distinta a la de los franceses. Zaragoza se quedó con 4,850 soldados para defender a la ciudad de Puebla. La tropa francesa llegó a las afueras de Puebla a las 9 a.m. el 5 de mayo. El general Latrille y su ejército intentó tomar la ciudad de Puebla tres veces pero la determinación y el esfuerzo de los defensores mexicanos no lo permitió y los franceses retrocedieron. El pequeño ejército mexicano por fin había vencido al invencible ejército francés. Aunque un año después los franceses pudieron vencer al gobierno mexicano, el Cinco de Mayo simboliza un México unido en favor de la Patria.

\section{La expansión}

Fue el valor de los mexicanos en el triunfo de Puebla lo que llevó a inspirar el orgullo en los mexicoamericanos. Aunque se considera un día festivo en México, el Cinco de Mayo se ha reducido a una celebración en ciudades como Puebla y México. Pero en los EE.UU., según Alvar W. Carlson, se realizan más de 120 festivales del Cinco de Mayo en por lo menos 21 estados, entre los que están Oregón, Chicago y Hawái. También se encuentra la presencia del Cinco de Mayo en lugares adonde no se suele pensar que llegaría una fiesta mexicana. Tal es el caso de Cheyenne, Wyoming donde la emigración agrícola ha llevado a los mexicoamericanos a establecerse (Carlson 10). En el norte del país se encuentra una población mexicoamericana concentrada principalmente en la industria y la producción. Por ejemplo, en Detroit, Michigan la población de origen mexicano era de 616, 300 en 1990, y el primer festival del Cinco de Mayo se realizó en 1985 (Carlson 10). Pero fue en el suroeste del país, en el estado de California, donde el Cinco de Mayo empezó a mostrar su presencia justo un año después de la batalla de Puebla.

\section{El resultado}

Cuando los franceses se retiraron de Puebla, los mexicanos se regocijaron tras haber defendido a su tierra, y la Batalla fue allanando el camino hacia un encuentro con lo mexicano. Esta noticia tardo en llegar a los EE.UU., donde se encontraban los "mexicanos aislados". El ejemplo se encuentra con un grupo de hijos del "otro lado", los californios, quienes no habían escuchado la tremenda noticia hasta la última semana de mayo (Hayes-Bautista y Chamberlin 23). Este grupo fue forzado a reconocerse como ciudadanos 
de los EE. UU. cuando se realizó el Tratado de Guadalupe Hidalgo el 2 de febrero de 1848. Para colmo, las escrituras de propiedad de los californios fueron considerados nulos y sin valor por el Consejo de Tierras en California. Como los californios fueron oprimidos por los americanos durante esta época, el hecho de que México (un país con menos recursos) venciera a otro extranjero produjo en estos mexicoamericanos una identidad colectiva y un nexo con sus hermanos, quienes habían salvado la República por el momento, por lo que también se sintieron vencedores. Al escuchar a sus compatriotas en Puebla, un grupo de californios en San Francisco formó juntas patrióticas cuya idea era extender apoyo moral y, en algunos casos, recaudar fondos para el gobierno de Benito Juárez, el presidente de México en ese tiempo. Las juntas realizaban desfiles militares con las banderas de México y los EE.UU. Los hogares eran decorados y los encargados preparaban un discurso para resumir el significado del día y detallar los acontecimientos que tuvieron lugar (Hayes-Bautista y Chamberlin 24). Aunque esta población de californios fue desplazada por tratados legales, sus raíces no se rompieron de la tierra y, como eslabones, la gente quedó unida a México.

\section{Los chicanos}

Los veteranos de la primera oleada de participantes de los festivales en San Francisco, según mencionan David E. Hayes-Bautista y Cynthia L., habían inculcado el Cinco de Mayo en las generaciones subsiguientes como "una memoria vívida que los latinos [no permitirán perder]" (47). Al correr los años, la escena de conmemoraciones del Cinco de Mayo en California fue cambiando. No fue hasta la llegada de otros movimientos sociales como el de los derechos civiles después de la Segunda Guerra Mundial, que los chicanos llegarían en los años 60 para buscar un día simbólico para sus esfuerzos. En su artículo, "Symbols and Style in Cinco de Mayo", Laurie Kay Sommers considera que el uso del Cinco de Mayo por parte de los chicanos es una herramienta que "simboliza una experiencia chicana bicultural que es algo más, algo reinterpretado y reestructurado [de lo original]" (478). Como los mexicanos usaron el Cinco de Mayo como un símbolo de la resistencia contra el extranjero, los chicanos lo usarían como resistencia contra los EE.UU., particularmente contra las leyes que discriminaban a la raza al tiempo que denunciaban las precarias condiciones de vida en sus comunidades. Según Laurie Kay Sommers, "los activistas tenían en cuenta que el significado histórico del Cinco de Mayo entre México era un símbolo de la autodeterminación [de los pobladores]" y lo escogieron como el día festivo para los chicanos (Sommers 1991, 479). Algunos de los elementos que este grupo de latinos empezó a añadir a la celebración fueron exhibiciones de autos lowrider, ballets folklóricos, danzas indígenas, murales y discursos promoviendo el progreso de los chicanos. Lo interesante de este grupo en California fue que usaron iconos políticos como César Chávez y la cultura de los barrios. En fin, los chicanos encontraron un símbolo de fuerza y resistencia pero también, y de manera importante, de la recién nacida identidad chicana.

\section{La Raza Unida}

Como se mencionó anteriormente, la expansión de la celebración del Cinco de Mayo ha llegado a incluir varios países en diferentes orillas de los EE.UU. En algunos de estos lugares se encuentra una dinámica cultural mezclada que se ha unido al Cinco de Mayo para representar una declaración de orgullo étnico 
y cultural. En la región de San Francisco (conocida como Bay Area), la integración de aspectos de otras comunidades latinas comenzó cuando la migración de una ola de centroamericanos llegó en los años 80 (Sommers 1991, 43). En los festivales de esa década, las formas de expresión incluyeron bailes de diferentes países latinoamericanos y la integración de música como salsa y la nueva canción. La presencia de una raza que no era mexicana pero unida, ya ocurría en el oeste de los Estados Unidos desde hacía mucho tiempo. David E. Hayes-Bautista y Cynthia L. Chamberlin, indican que el área de California después de 1848 había atraído a muchos grupos de gente por el Gold Rush (la "fiebre del oro") que incluía a inmigrantes de Chile, Colombia, Perú y Centro América (39). En uno de los discursos sobre la conmemoración del Cinco de Mayo en 1864, en Los Ángeles, se afirma que el público incluía "mexicanos, californios, norteamericanos, y españoles-americanos que representaban casi todas las repúblicas de América" (Hayes-Buatista y Chamberlin 45). Un simpatizante de descendencia colombiana explicó que él apoyaba a México y protestaba contra el gobierno francés, "[México], te saludo y te felicito en este día alegre y memorable, el Cinco de Mayo, el cual celebramos su aniversario." (Hayes-Bautista y Chamberlin 46). Se sentían vinculados a la historia de Puebla como si fuera versión moderna de David y Goliat. Los latinos estaban unidos por la lengua, la simpatía y la identidad de intereses.

Algunos estadounidenses perciben al Cinco de Mayo como un St. Patrick's Day donde no se requiere ser irlandés para celebrar. Aunque se encuentran ofertas de descuento para cervezas en los restaurantes y cualquier participante puede conseguir sombrero y bigote sintético para festejar, es importante reconocer que la historia detrás del Cinco de Mayo es más profunda. El día representa el ímpetu para seguir adelante junto con la resistencia contra los opresores de los mexicanos, mexicoamericanos y otros latinos. Es un día que llegó a simbolizar el nacionalismo mexicano, un encuentro de un hermano con el otro que se unieron por La Causa y a la vez muestra el afecto hacia la madre patria. 


\section{Bibliografía}

Cabello-Argandoña, Roberto. Cinco De Mayo: A Symbol of Mexican National Resistance. Ed. Luis A. Torres. Encino: Floricanto, 1997. Impreso.

Carlson, Alvar W. "America's Growing Observance of Cinco de Mayo”. Journal of American Culture 21.2 (1998): 7-16. Web. Wiley Online Library. 20 Abril 2016.

Hayes-Bautista, David E., and Cynthia L. Chamberlin. "Cinco De Mayo's First Seventy-Five Years in Alta California: From Spontaneous Behavior to Sedimented Memory, 1862 to 1937". Southern California Quarterly 89.1 (2007): 23-64. JSTOR. Web. 2 Abril 2015.

Sommers, Laurie Kay. "Symbol and Style in Cinco de Mayo". The Journal of American Folklore 98.390 (1985): 476-462. JSTOR. Web. 1 Abril 2015.

Sommers, Laurie Kay. "Inventing Latinismo: The Creation of 'Hispanic' Panethnicity in the United States”. The Journal of American Folklore 104.411 (1991): 32-53. JSTOR. Web. 1 Abril 2015. 